\title{
A Case Series of Hemichorea due to Non Ketotic- Hyperglycemia with Unique MRI Brain Finding
}

\section{A K Roy*, Nadig Raghunandan, GRK Sarma and Thomas Mathew}

Department of Neurology, St Johns' Medical College Hospital, Bangalore, Karnataka, India

\begin{abstract}
Hemiballismus- hemi chorea (HB-HC) is an uncommon disorder, mostly seen among the people in Asian region. Recent reports have revealed that it is associated with non-ketotic hyperglycemia. MR spectroscopy in the present study showed a low NAA/Cr, suggesting pronounce energy depletion and neuronal dysfunction. Scientists observed that unilateral lesions occurred in elderly diabetic patients in striatum due to hyperglycemia. Our study reveals that, dyskinesia in elderly diabetic patients leads to an initial manifestation of diabetes among the both sexes.
\end{abstract}

Keywords: Hemichorea; MRI; Non ketotic-hyperglycemia

\section{Introduction}

Hemi chorea generally occurs due to a stroke, neoplasm or granuloma in the contra lateral basal ganglia [1]. Metabolic derangements especially hyperglycemia can also cause focal neurological dysfunction like hemiparesis, focal seizure [2,3]. Recently there have been case reports of hemichorea reported due to non-ketotic hyperglycemia with characteristic MRI findings predominantly in the Asian population [4]. The aim of this study is to highlight the important aspects pertaining to the clinical and neuroradiological diagnosis of hemichorea associated with nonketotic hyperglycemia.

\section{Materials and Methods}

Patients admitted with acute hemichorea were included for the study. A detailed history and examination was done in all patients. Hemogram, Blood sugar, serum electrolytes, serum calcium, HbA1C, liver function test, renal function test, lipid profile and MRI of the brain were done.

\section{Results}

From May 2008 to September 2009 there were 9 patients admitted with hemichorea. 3 patients (33\%) were found to have acute striatal infarcts, 2patients (22\%) had granuloma and other four patients(45\%) had hyperglycaemia without ketosis.

The median age of these 4 patients who had hyperglycemia was 60 years (58 to $70 \mathrm{yrs}$ ), male to female ratio was 1:1.All were right handed persons. 3 patients were known diabetic, their mean duration of diabetes was 12 years. 1 patient was detected to be diabetic at admission. None of these patients had any history of cerebrovascular event in the past.

All these patients presented with an acute history of involuntary jerky movements involving one side of the body, mean duration of symptom was 2 days. All had left sided chorea with normal cognition and motor power in the limbs. All had high blood sugars with mean of $318 \mathrm{mg} / \mathrm{dl}(252-475 \mathrm{mg} / \mathrm{dl})$ and mean HBA1c of 11.2. None had ketone bodies in the urine. Serum electrolytes, Calcium, blood urea nitrogen, creatinine and Liver function tests were normal.

Neuroimaging of these patients with MRI revealed unilateral hyper intensity in Straitum in T1W images contralateral to the side of hemichorea with mild hypointensity in T2 flair. There was no restriction in diffusion weighted images and MRS showed decreased NAA peak (Figure 1).
Chorea subsided once sugars were controlled, average duration was 4 days.

\section{Discussion}

Hemiballismus- hemi chorea (HB-HC) is an uncommon disorder, a vascular lesion being the most common cause [1]. More recently there have been reports of $\mathrm{HBHC}$ associated with non-ketotic hyperglycemia amongst people of Asian origin [3]. In our study nonketotic hyperglycemia was the commonest cause for hemichorea. This could be due to the high prevalence of diabetes mellitus which was 62.4 million in 2011 [5] and estimated to increase to 100 million by 2030 [6]

Dubinsky et al. first reported two patients with non-ketotic hyperglycemia with neuroimages that demonstrated a faint increase in densities over the contralateral basal ganglia [7]. Later, Yahikozawa et al. suggested that a combination of HB-HC and striatal hyperintensity on T1- weighted MRI in diabetic patients may constitute a unique syndrome [8]. A study by Lee et al. reviewed the characteristic clinicoradiologic syndrome and suggested that it was benign [9].

Previous studies [3] revealed that this dyskinesia often occurred in elderly diabetic patients and could also be an initial manifestation of diabetes with equal incidence among both sexes. In our study one patient was newly detected with diabetes on admission. The incidence among both sexes was equal.

In MRI T1 weighted images showed hyperintensity signals in the contralateral striatum. The mechanism of this characteristic signal changes on MRI is still debated. In view of the acute clinical manifestation and the striatal lesion corresponding to the territory of lenticulostriate artery, it has been suggested that the lesion may be secondary to vascular insult. Based on the evolution of clinical manifestation and the findings of the neuroimages, Chang et al. [10] suggested that petechial hemorrhage in the putamen might be the pathological mechanism to cause the dyskinesia. Moreover, studies of

*Corresponding author: A K Roy, Department of Neurology, St Johns' Medical College Hospital, Bangalore, India, E-mail: rroy_ajit@hotmail.com

Received May 03, 2013; Accepted June 11, 2013; Published June 20, 2013

Citation: Roy AK, Raghunandan N, Sarma GRK, Mathew T (2013) A Case Series of Hemichorea due to Non Ketotic- Hyperglycemia with Unique MRI Brain Finding. J Neurol Neurophysiol S11:006. doi:10.4172/2155-9562.S11-006

Copyright: ( 2013 Roy AK, et al. This is an open-access article distributed under the terms of the Creative Commons Attribution License, which permits unrestricted use, distribution, and reproduction in any medium, provided the original author and source are credited. 
Citation: Roy AK, Raghunandan N, Sarma GRK, Mathew T (2013) A Case Series of Hemichorea due to Non Ketotic- Hyperglycemia with Unique MRI Brain Finding. J Neurol Neurophysiol S11:006. doi:10.4172/2155-9562.S11-006

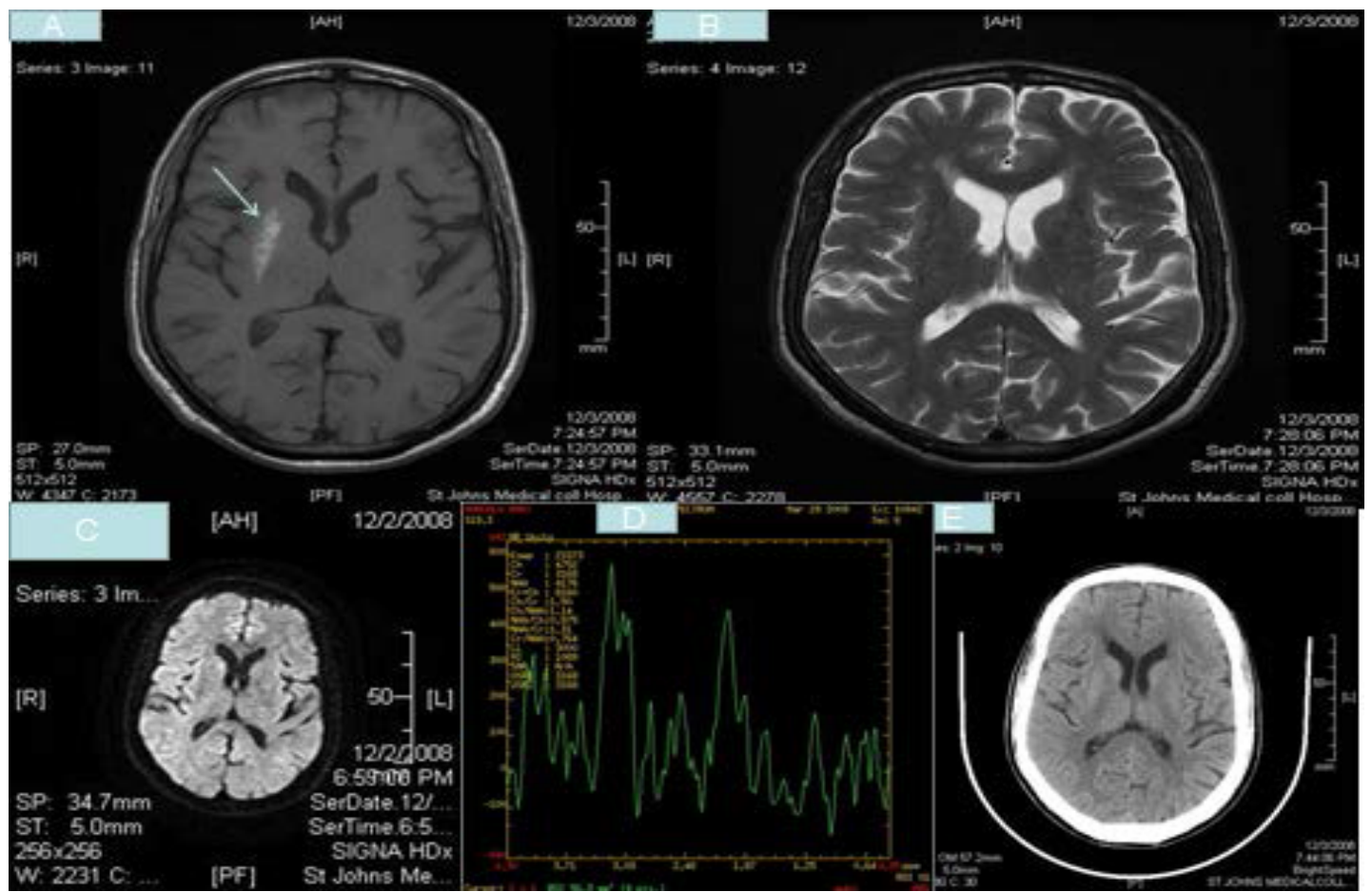

Figure 1: T1W axial image (A) showing striatal hyperintensity(arrow),T2W axial images(B) showing hypointensity, Diffusion weighted images(C) showing no restriction, magentic resonance spectroscopy(D) showing reduced NAA peak and CT axial images(E) showing hyperdensity in striatum (MRI was done by General Electric HBX, 1.5 Telsa machine, 2007 model with 4.3 version software).

Altafullah et al. and Broderick et al. suggested that the lesions might have resulted from hemorrhagic insult [11,12]. However, a biopsy specimen from the hyperintense putamen revealed only a slight astrocytosis and vacuolization or a fragment of gliotic brain tissue with abundant gemistocytes with no deposition of hemosiderin [13].

MR spectroscopy in the present study showed a low NAA/Cr, suggesting pronounce energy depletion and neuronal dysfunction. This is secondary to neuronal loss or damage either due to hypoperfusion or metabolic failure. Studies by SPECT [14,15] and PET [16] have revealed the reduction of blood flow and metabolism in the contralateral striatum of patients with HB-HC caused by non-ketotic hyperglycemic.

The probable hypothesis as proposed by Juei-Jueng Lin et al. is that the metabolic derangements (hyperglycemia) and cerebral vascular insufficiency both contributed to regional metabolic failure in basal ganglion cells [17].

Proper functioning of basal ganglia has been compromised by chronic ischemia through microangiopathic changes, even though an actual infarct has not yet developed. When a stage of metabolic crisis is achieved, either there is further compromise of circulation and glucose hypometabolism, which precipitates the clinical syndrome of hemichorea $[17,18]$. This process triggers reactive astrocytosis in the basal ganglia and probably enhances local manganese accumulation and thus produces the typical signal changes seen on MRI [17].

\section{Conclusion}

Unilateral lesions can occur in Striatum due to hyperglycemia in elderly diabetic patients, which is hyperintense in T1W images. Recognition of this unique clinicoradiologic manifestation is important because correction of the underlying hyperglycemia will lead to rapid improvement.

\section{References}

1. Dewey RB Jr, Jankovic J (1989) Hemiballism-hemichorea. Clinical and pharmacologic findings in 21 patients. Arch Neurol 46: 862-867.

2. Maccario M (1968) Neurological dysfunction associated with nonketotic hyperglycemia. Arch Neurol 19: 525-534.

3. Grant C, Warlow C (1985) Focal epilepsy in diabetic non-ketotic hyperglycaemia Br Med J (Clin Res Ed) 290: 1204-1205.

4. Oh SH, Lee KY, Im JH, Lee MS (2002) Chorea associated with non-ketotic hyperglycemia and hyperintensity basal ganglia lesion on T1-weighted brain MRI study: a meta-analysis of 53 cases including four present cases. J Neurol Sci 200: 57-62.

5. Anjana RM, Pradeepa R, Deepa M, Datta M, Sudha V, et al. (2011) Prevalence of diabetes and prediabetes (impaired fasting glucose or/and impaired glucose tolerance) in rural and urban India: Phase 1 results of the Indian Council of Medical Research-INdiaDIABetes (INDIAB) study. Diabetologia 54: 3022-3027.

6. Unwin N, Whiting D, Guariguata L, Ghyoot G, Gan D (2011) In Diabetes Atlas (5thed) Belgium: International Diabetes Federation.

7. Dubinsky RM, Greenberg M, Di Chiro G, Baker M, Hallett M (1989) Hemiballismus: study of a case using positron emission tomography with 18fluoro-2-deoxyglucose. Mov Disord 4: 310-319.

8. Yahikozawa H, Hanyu N, Yamamoto $\mathrm{K}$, Hashimoto $\mathrm{T}$, Shimozono $\mathrm{K}$, et al. (1994) Hemiballism with striatal hyperintensity on T1-weighted MRI in diabetic patients: a unique syndrome. J Neurol Sci 124: 208-214.

9. Lee BC, Hwang SH, Chang GY (1999) Hemiballismus-hemichorea in older diabetic women: a clinical syndrome with MRI correlation. Neurology 52: 646648

10. Chang MH, Chiang HT, Lai PH, Sy CG, Lee SS, et al. (1997) Putaminal petechial haemorrhage as the cause of chorea: a neuroimaging study. J Neurol Neurosurg Psychiatry 63: 300-303.

11. Altafullah I, Pascual-Leone A, Duvall K, Anderson DC, Taylor S (1990) Putaminal hemorrhage accompanied by hemichorea-hemiballism. Stroke 21 1093-1094. 
Citation: Roy AK, Raghunandan N, Sarma GRK, Mathew T (2013) A Case Series of Hemichorea due to Non Ketotic- Hyperglycemia with Unique MRI Brain Finding. J Neurol Neurophysiol S11:006. doi:10.4172/2155-9562.S11-006

Page 3 of 3

12. Broderick JP, Hagen T, Brott T, Tomsick T (1995) Hyperglycemia and hemorrhagic transformation of cerebral infarcts. Stroke 26: 484-487.

13. Ohara S, Nakagawa S, Tabata K, Hashimoto T (2001) Hemiballism with hyperglycemia and striatal T1-MRI hyperintensity: an autopsy report. Mov Disord 16: 521-525.

14. Belcastro Vincenzo, Pierguidi Laura, Tambasco Nicola, Sironi Luigi, Sacco Leonardo, et al. (2011) Decreased Contralateral Putamen [I]FP-CIT SPECT Uptake in Hyperglycemic Hemichorea-Hemiballismus. European Neurology May 65: 307-308.

15. Chang MH, Li JY, Lee SR, Men CY (1996) Non-ketotic hyperglycaemic chorea: a SPECT study. J Neurol Neurosurg Psychiatry 60: 428-430.
16. Hsu JL, Wang HC, Hsu WC (2004) Hyperglycemia-induced unilateral basal ganglion lesions with and without hemichorea. A PET study. J Neurol 251 1486-1490.

17. Lin JJ, Lin GY, Shih C, Shen WC (2001) Presentation of striatal hyperintensity on T1-weighted MRI in patients with hemiballism-hemichorea caused by nonketotic hyperglycemia: report of seven new cases and a review of literature. $J$ Neurol 248: 750-755

18. Battisti Carla, Forte Francesca, Rubenni Elisa, Dotti Maria Teresa, Bartal Anna, et al. (2009) Two cases of hemichorea-hemiballism with nonketotic hyperglycemia: a new point of view. Neurol Sci 3: 179-183. 